Gdańsk 2020, Nr. 43

https://doi.org/10.26881/sgg.2020.43.04

Marek Cieszkowski

Universität Bydgoszcz / Uniwersytet Kazimierza Wielkiego w Bydgoszczy

ORCID: 0000-0003-3170-9867

\title{
Unter dem Zeichen von Hammer, Sichel und Weizenährenkranz - wolgadeutscher Sprachgebrauch in den 20er- und 30er-Jahren des 20. Jh.
}

Im Mittelpunkt des Interesses steht die geschriebene Varietät der deutschen Sprache, die in der wolgadeutschen Presse des 20er- und 30er-Jahre des 20. Jh., d. h. in der Zeit, in der sie unter starkem Einfluss kommunistischer Ideologie und Parteipropaganda stand, belegt ist. Qualitative Aussagen über die Russlanddeutschen werden mit Paradigmenwechsel in der modernen Wissenschaft assoziiert und mit der Politik der Perestroika und Glasnost bezeugt, die es erst möglich machte, russische Archive in den 80er- und 90er-Jahren des 20. Jh. zu öffnen.

Schlüsselwörter: Wolgadeutsch, Presseartikel, kommunistische Ideologie, Parteipropaganda

With a hammer, a sickle, and a wreath made of ears of wheat - the language of Germans of the Volga Region in the second and third decade of the $20^{\text {th }}$ century. - The paper focuses on the written variant of German as used by the Germans of the Volga Region in their press in the second and third decade of the $20^{\text {th }}$ century. The study of its unique character shaped by communist ideology and one-party propaganda has been combined with a qualitative change in the study of Russian Germans and with the data coming from the Russian archives opened as a result of glasnost and perestroika in the ' 80 s and the ' 90 s of the last century.

Keywords: Volga German, press articles, communist ideology, one-party propaganda

Deutsche Siedler in Russland ist kein neues Thema für die Wissenschaft. Historisch, aber auch politisch sowie kultur- und gesellschaftskritisch gesehen. Bevor ich mich mit einigen neueren Aspekten dieser Problematik befasse, möchte ich stichwortartig auf ausgewählte Stationen der deutschen Siedlungsgeschichte in Russland eingehen, um zunächst einmal das geschichtlich vermittelte Bild der Russlanddeutschen ins Gedächtnis zu rufen, die Geschichte bisheriger russlanddeutscher Forschungen typologisch darzustellen und für eine sprachwissenschaftliche Darstellung, den Sprachgebrauch der Wolgadeutschen in den 20er- und 30er-Jahren des 20. Jh., als verwertbar festzuhalten. Bei der Darstellung, in der historisch-kulturelle und strukturelle Aspekte im Vordergrund stehen, wird auf die Entwicklungstendenzen des wolgadeutschen Wortschatzes aus der Sicht der synchronen Wortbildung eingegangen. 


\section{Anhaltspunkte der russlanddeutschen Geschichte}

Deutsche Siedler gelangten bereits im 16. Jh. ins Zarenreich und als geschätzte Experten auf dem Gebiet der Kriegskunst, der Verwaltung, der Medizin und der Technik (vgl. Dietz / HiLKes 1992: 13) gehörten sie zur gesellschaftlichen Elite. In der 2. Hälfte des 18. Jh. kamen erneut etwa 27.000 Siedler aus deutschen Landen, diesmal aber zumeist bäuerlicher Herkunft, in die Wolgagebiete, wo die Zarenregierung unter Katharina II. (1762-1796) ihnen im Manifest von 22. Juli 1763 einige wichtige Privilegien versprach. Diese Privilegien, darunter vor allem Religionsfreiheit, dreißig Jahre Steuer- und Abgabenfreiheit, zinsloses Darlehen zum Aufbau der Bauernwirtschaft oder des Handwerksbetriebes, Selbstverwaltung sowie Befreiung vom Militär- und Zivildienst, waren Versprechungen, die nicht oder nur teilweise erfüllt wurden; die eingewanderten Bauern wurden gezwungen, unter sehr harten Lebensund Arbeitsbedingungen ihre neue Existenz an beiden Seiten der Wolga (vgl. BRANDES 1997: 57; Lebioda 2004: 74; SinNer 1923:3) aufzubauen. Parallel dazu sind in der selben Zeit neue deutsche Siedlungen um St. Petersburg und im Schwarzmeergebiet entstanden (vgl. Dietz / Hilkes 1992: 15).

Deutsche Kolonisten gelangten nach Russland auch am Anfang des 19. Jh. unter Alexander I. (1801-1825) und errichteten in Gebieten der heutigen Ukraine, der Krim, des Transkaukasus und Bessarabiens weitere Siedlungen; am Ende des 19. Jh. gab es neue Siedlungen in Sibirien, Kasachstan und Mittelasien; laut zugänglichen Statistiken wurden in den Jahren 1763-1914 auf dem russischen Territorium mehr als 3000 deutsche Kolonien gegründet (vgl. Dietz / Hilkes 1992: 15).

In der 2. Hälfte des 19. Jh. beginnen erste Abwanderungen, die vor allem mit der sozialen und politischen Situation in den deutschen Siedlungsgebieten in Russland zusammenhängen; man nahm die im Zarenmanifest zugesicherten Privilegien teilweise zurück, behandelte danach deutsche Siedler wie russische Bürger und zwang zum Militärdienst; verheerende Missernten im Wolgagebiet waren weitere Abwanderungsgründe ins Ausland und nach Mittelasien.

Der I. Weltkrieg brachte wesentliche Veränderungen für die Russlanddeutschen mit sich. Im Jahre 1915 wurden deutsche Siedler als Angehörige einer feindlichen Nation bezeichnet und in den Osten deportiert. Weitere Deportationen wurden verhindert, als 1917 die Oktoberrevolution ausbrach und 1918 der Bürgerkrieg folgte; Wolgadeutsche erlebten schwere Notzeiten.

Wolgadeutsche wie alle Russlanddeutschen profitieren am Anfang der 20er-Jahre von der zunächst relativ liberalen, nationalitätenfördernden Politik (vgl. CIEszKowski 2015: 47), nach der sie Einfluss auf das gesellschaftspolitische Geschehen gewinnen und stärker an den neuen Staat gebunden werden. Ein gutes Beispiel dazu ist das bereits im April 1918 in Saratow errichtete Kommissariat für deutsche Angelegenheiten, dessen Aufgabe in der Übergangszeit es war, sämtliche Verwaltungsaufgaben in den wolgadeutschen Kolonien zu erledigen und die Kolonisten in Kontakten mit russischen Behörden zu unterstützen (vgl. SCHULZEMölKaU (1931: 41-43). Ab Ende der 20er-Jahre kommt es allerdings „zur gewaltsamen Unterdrückung nationaler Bestrebungen“ sowie zum „Abbau von nationalitätenpolitischen 
Zugeständnissen und der Liquidierung nationaler Kader" (DeIBERT 2009: 11). Seit der Machtergreifung von Hitler wird die Lage der Deutschen in dem Sowjetstaat noch zusätzlich dadurch erschwert, dass sie „im Spannungsfeld zweier miteinander verfeindeter totalitärer Systeme“" (DeIBert 2009: 7) lebten.

Auf der Delegiertenversammlung in Warenburg, die vom 24.-28. Februar 1918 stattfind, wurde die Errichtung einer Autonomen Föderation an der Wolga beschlossen, die im Februar 1924 zur Autonomen Sozialistischen Sowjetrepublik der Wolgadeutschen ${ }^{2}$ (ASSW) erhoben wurde (vgl. Dietz / Hilkes 1992: 18)33. Sie bestand bis zum 27. August 1941, als auf Befehl des Volkskommissars für Innere Angelegenheiten, Lawrenti Beria, die Deutschen aus der Republik der Wolgadeutschen, den Gebieten Saratow und Stalingrad (präventiv) ${ }^{4}$ umgesiedelt wurden.

Das damalige wolgadeutsche Staatswesen symbolisiert das Staatswappen, auf dem eine goldene Sichel und ein goldener Hammer in den Strahlen der Sonne zu sehen sind, umgeben von einem Weizenährenkranz und mit einer deutschen und russischen Aufschrift in der rechten und linken Ecke versehen: Proletarier aller Länder vereinigt euch.

\section{Zur Typologie bisheriger Forschungen zu Russlanddeutschen}

In der Fachliteratur wird oft in Bezug auf die Forschungen zur russlanddeutschen Geschichte von vier verschiedenen Zeitperioden gesprochen (vgl. Neutatz 2016: 595-612). Die erste Periode, die im 19. Jh. beginnt und mit dem 1. Weltkrieg endet, wird pastoral genannt. Es geht dabei um Schreibleistungen von russlanddeutschen Pastoren und Pfarrern, deren Tätigkeit vor allem darin bestand, „Quellen [zu] dokumentieren, die heute nicht mehr erhalten oder zugänglich sind“" (NeutaTZ 2016: 597). Die Geistlichen haben überdies Texte geschrieben, die thematisch gesehen homogen waren und grundsätzlich kirchliche und religiöse Probleme der damaligen Zeit zum Gegenstand hatten.

Die zweite Periode erstreckt sich auf die Zwischenkriegszeit und wird gewöhnlich mit zwei Adjektiven beschrieben: akademisch und nationalistisch (1. akademische Periode). In dieser Zeit werden erste umfassende Studien geführt, deren Autoren russlanddeutsche Studenten und künftige Akademiker sind, die in Deutschland studiert und an deutschen Universitäten gewirkt haben. Die akademische Periode schlägt nach 1933 nach der Machtergreifung von Hitler in eine nationalistische Periode um und wird mit den Oststudien in Verbindung gesetzt, die staatlich gefördert wurden. Die Qualität der wissenschaftlichen Texte ist verschieden,

\footnotetext{
Zu Beschlüssen der Delegiertenversammlung vgl. SChulze-MölkaU (1931: 32).

Über die Hintergründe der Umwandlung der ursprünglichen Arbeitskommune in die Wolgarepublik ist u. a. bei SCHULZE-MÖLKAU (1931: 43-51 und 52) nachzulesen.

3 Vgl. dazu die Begründung von Schulze-Mölkau (1931: 53): „Der Geburtstag der Wolgarepublik ist [...] der 20. Februar 1924, an dem die spontane Äußerung des Gebietsrätekongresses die staatliche Sanktion erhält“.

4 In der Fachliteratur werden dabei zwei entgegengesetzte Meinungen präsentiert. Einerseits wird angenommen, dass die stalinistischen Deportationen als „eine Präventivmaßnahme und somit als Folge des Krieges“ anzusehen sind, andererseits wird darauf verwiesen, dass sie „als Teil des Konzepts der stalinistischen Nationalitätenpolitik“ (DEIBeRt 2009: 10 und 11) innerhalb des stalinistischen Systems zu betrachten sind.
} 
in vielen Fällen kann jedoch von einem Fortschritt in Bezug auf die behandelte Thematik und methodisch-methodologische Ausrichtung gesprochen werden. Etliche Texte sind im Rahmen der damals betriebenen Volksgeschichte entstanden, die heute „als eine der Wurzeln der späteren modernen Sozialgeschichte" (NeUTATz 2016: 597) angesehen wird.

Für die dritte Periode werden ebenfalls zwei zusammengesetzte Adjektive benutzt, mit denen diverse Aktivitäten zu Russlanddeutschen in der Zeit nach dem 2. Weltkrieg bis in die 70er-Jahre des 20. Jh. thematisiert werden: landsmannschaftlich und heimatkundlich. Die Beschäftigung mit den deutschen Minderheiten galt als verpönt oder wurde marginalisiert; etliche Materialien über das Schicksal der Russlanddeutschen (Textkorpora) sind durch den Krieg verlorengegangen, dazu war der Zugang zu Archiven in der Sowjetunion erschwert oder überhaupt nicht möglich. Auch in der ehemaligen DDR hat man das Phänomen der deutschen Minderheiten trotz besserer Informationsmöglichkeiten eher gemieden'5 Auf ähnliche Weise hat die sowjetische Forschung gehandelt ${ }^{6}$. Wie Neutatz (2016: 597) feststellt, „an die Stelle der wissenschaftlichen Forschung trat für einige Jahrzehnte sowohl in Deutschland als auch in Amerika das landsmannschaftlich-heimatkundliche Schrifttum". Es ging in erster Linie darum, die Erinnerung an die Auswanderer wachzuhalten und ihre Lebensweisen zu belegen.

Um die Wende der 70er- und 80er-Jahre meldet sich eine junge Forschergeneration zu Wort und beginnt neue Akzente in der russlanddeutschen Forschungsgeschichte zu setzen. Es sind Historiker und Vertreter von benachbarten Disziplinen, die sich von einer großgeschriebenen Geschichte im staats- und sozialpolitischen Sinn abwenden und sich allmählich zugunsten einer Alltagsgeschichte von Menschen zuwenden. $\mathrm{Zu}$ betonen ist in diesem Zusammenhang die Politik der Perestroika und Glasnost in der Sowjetunion, die es erst dann möglich machte, sowjetische Archive zu öffnen und jahrzehntelang gemiedene Probleme neu (kulturell, national, sozial und historisch) zu perspektivieren ${ }^{7}$. Diese Periode wird als akademisch (2. akademische Periode) bezeichnet und dauert eigentlich bis heute an. In den 90er-Jahren wird in der Forschung von einer „Enttabuisierung der sowjetdeutschen Problematik in den Nachfolgestaaten der UdSSR“" (Deibert 2009: 9) gesprochen. Auch ein Teamforschungsprojekt zur Sowjetisierung der wolgadeutschen Sprachvarietät ${ }^{8}$, an dem ich

Man findet wenige Gegenbeispiele, vgl. z. B. JEDIG (1986: 74-80).

Vgl. die Aussage von Dietz / HiLkes (1992: 9): „Bis in die achtziger Jahre hinein ließ sich die aktuelle Situation der Deutschen in der Sowjetunion nur durch spärliche und verstreute Meldungen in sowjetischen Publikationen - vornehmlich in den deutschsprachigen - erfassen“.

In dieser Zeit kamen „die stenographischen Protokolle der Zentralkomiteesitzungen, der Schriftverkehr zwischen dem zentralen Parteiapparat und den Komitees in den Provinzen, die Korrespondenz und die Aktenführung der Staatsbehörden und die Papiere Stalins, Molotows, Kaganowitschs und anderer politischer Führer" (BABEROwSKI 2014: 22) ans Tageslicht, die eine systematische, skrupellos betriebene Vernichtungspraxis und Gewaltexzesse im inneren Kreis der kommunistischen Machthaber dokumentieren.

8 Es handelt sich um das Projekt Zur Sowjetisierung der wolgadeutschen Sprachvarietät in den 20erund 30er-Jahren, das durch Finanzmittel des Polnischen Zentrums für Wissenschaft (DEC-2012/ 05/B/ HS2/04104) gefördert wurde. Die Forschungsergebnisse wurden in drei Bänden präsentiert; vgl. MĘDELSKA / Cieszkowski / Jankowiak-Rutkowska (2017); MęDelska / Cieszkowski / Sobczak (2017); CieszKOWSKI (2017). 
mit meinen KollegInnen in den Jahren 2013-2017 gearbeitet habe, hätte ich früher nicht realisieren können.

Eine neue Schwerpunktsetzung ist nicht zufällig, denn gerade zu Beginn der 80er-Jahre zeichnet sich in modernen Kulturwissenschaften eine Tendenz ab, nach der „mikrohistorische Erforschung von Alltag, individuellen und kollektiven Lebenswelten und Mentalitäten" (NEUTATZ 2016: 603) das etablierte Forschungsparadigma besetzt. Man will nicht „mit abstrakten Theorien arbeiten, sondern Erfahrungen rekonstruieren“, da „abstrakte Begriffe nicht in der Lage seien, die qualitativen Aspekte menschlichen Denkens ohne Verlust und Verzerrung zu verstehen und zu vermitteln". Gefordert wird eine intensive, andersgeartete Quellenarbeit, in der in den Mittelpunkt des Interesses Menschen gestellt werden, „die nicht an den Hebeln der Macht standen" (NeutaTZ 2016: 604), sondern in ihrem Alltag Geschichten erlebt haben, in denen die politische Macht nur ein Bestandteil ihrer komplizierten Erfahrungen war. Mit solch einer Perspektivierung wird ein neues und ertragreiches Forschungsfeld eröffnet.

Eine besondere Rolle kommt dadurch der Sprache zu. Einerseits werden in ihr und mit deren Hilfe unsere Erfahrungen, Werthierarchien und soziale Praktiken in Texten fixiert, andererseits werden sie als Ergebnisse von kommunikativen Handlungen weitergegeben. Kurz gesagt: Sprache konstruiert das kommunikativ zu Vermittelnde. In Anlehnung daran lässt sich daher Folgendes feststellen: „man hat es nicht mehr mit der Realität an sich zu tun, sondern mit der Kommunikation über sie, mit sprachlichen Verhaltensmustern, der Art und Weise, wie Ereignisse oder Entwicklungsprozesse von den Zeitgenossen sprachlich thematisiert werden" (NeUTATZ 2016: 607); nicht zu vergessen ist dabei der Umstand, dass jegliche auf Quellen aufbauende Aussagen sprachliche Konstrukte sind. Aus diesem Grund scheint eine Reflexion über die Sprache, ihre Leistungen und Grenzen, unumgänglich zu sein.

\section{Wolgadeutscher Sprachgebrauch}

Wenn über den wolgadeutschen Sprachgebrauch gesprochen wird, da wird damit auf die Dialekte Bezug genommen, die die ehemaligen deutschen Einwanderer aus Hessen, Baden, der Pfalz, Lothringen und Elsass in der 2. Hälfte des 18. Jh. an die Wolga mitgebracht haben. Diese Dialekte (hessisch, pfälzisch, niederdeutsch und ostmitteldeutsch) dienten als homogenes Verständigungsmittel unter Angehörigen geschlossener Siedlergruppen, für deren Fortbestand landsmannschaftliche (vgl. BEREND 1998: 5) und familiäre Zugehörigkeit entscheidend waren; landsmannschaftliche Zugehörigkeit deckte sich in diesem Fall nahezu ausnahmslos mit der konfessionellen Zugehörigkeit (vgl. Bohmann 1970: 66). Somit bestand innerhalb der Gruppen eine sozial-kulturelle und religiöse Homogenität.

Die wolgadeutschen Siedler kamen auch mit einer standardnahen Variante der deutschen Hochsprache in Berührung, die in konfessionellen Schulen vermittelt wurde; dazu ist noch die Sprache der kontaktierenden Umgebung (Russisch) zu erwähnen, die sich allmählich von einer Kontaktsprache zu einer „Überdachungssprache“ entwickelt hat (vgl. LÖFFLER 1994: 63).

Durch Kontakte verschiedener Art und Migrationsbewegungen kam es zu Mischungs- und Ausgleichstendenzen, die von Georg Dinges (1891-1932) und Andrej Dulson (1900-1973) im Rahmen der dialektologischen Untersuchungen in den 20er- und 30er-Jahren des 20. Jh. 
in der Zentralstelle für wolgadeutsche Mundartenforschung (Saratow) auf der Basis von Umfragen erfasst, eingeordnet und qualitativ sowie quantitativ ausgewertet wurden ${ }^{10}$. Ihre Erforschungen waren richtungweisend und haben für lange Zeit dialektologisch-ethnographische Studien zur russlanddeutschen Dialektologie im Allgemeinen und zur wolgadeutschen Dialektologie im Besonderen beeinflusst ${ }^{11}$.

Die moderne Forschung kann nur teilweise und nur noch in Bezug auf die geschriebene Sprache antworten, mit welcher Intensität und wie diese Mischungsprozesse verlaufen sind, ob die Basisdialekte ,in ihrer ursprünglichen Form erhalten geblieben sind oder ob sie sich in der neuen dialektalen Umgebung mit anderen Dialekten vermischt haben" (vgl. BEREND 1998, 10); als Forscher wird man vor Schwierigkeiten gestellt, die nicht nur aus rein technischen, sondern auch praktischen Gründen nicht zu lösen sind, wenn man die Entwicklungen in der gesamten russlanddeutschen Dialektlandschaft der letzten 100 Jahre berücksichtigt. So ist wohl die Idee verständlich, wenn man die dialektale Betrachtungsweise zugunsten einer aufgrund textueller Korpora aufbauenden, lexikologisch-lexikographischen Analyse in den Vordergrund stellt und sich auf qualitative und quantitative Studien konzentriert, in denen sprachlich repräsentierte, thematisch gegliederte Alltagserfahrungen der Wolgadeutschen von besonderer Relevanz sind.

\subsection{Zum sozialpolitischen und kulturellen Hintergrund}

Die 20er- und 30er-Jahre sind die Zeit, in der sich die bolschewistische Herrschaft entfaltet, und die (wolgadeutschen) Arbeiter- und Bauernmassen „einer brutalen Disziplinierungsund Erziehungsdiktatur" (BABEROwSKI 2014: 25) unterworfen wurden. Die sowjetische Ordnung, die ursprünglich als föderativ gedacht war, in der Tat aber nur Anzeichen des Föderalismus im westeuropäischen Sinn (vgl. SCHULZE-MÖLKAU (1931:38-40) trug, wurde für Millionen von Sowjetmenschen, darunter auch Wolgadeutschen, zu einem wesentlichen Teil ihrer totalitären Lebenserfahrung. Es ging darum, einen homogenen Kommunikationsraum zu schaffen, in dem das revolutionäre Vielvölkerreich seine Rituale als Möglichkeiten der Macht inszenierte: „Propaganda, Feste und Märsche, kulturrevolutionäre Umerziehungskampagnen und die Androhung von Terror und Gewalt"12 (BABEROwski 2014: 20). Eine wichtige Position im bolschewistischen Ordnungsprojekt nimmt der wirkliche, meistens aber der imaginäre, ideologisch definierte Feind ein, mit dem der kommunistisch regierte,

9 Über den Nachlass der Saratower dialektologischen Schule vgl. Jedig (2014: 155-166).

10 Vgl. dazu Dinges (1923: 60-72) und Dulson (1933: 46-54). Von Relevanz sind auch neuere Studien zur russlanddeutschen Dialektforschung; vgl. Domaschnew (1994: 165-177); Frank (1992); MosKALJUK (1998: 27-35).

11 Für die deutsche Dialektforschung dieser Periode hat sich auch Viktor Schirmunski verdienstlich gemacht, der Professor an der Leningrader Universität war und mit Georg Dinges nachweislich öfters im Kontakt stand. Vgl. Schirmunski, Viktor (1928): Die deutschen Kolonien in der Ukraine. Moskau.

12 Selbst die Stalinsche Verfassung (1936) diente nach ARENDT (2015: 826) dazu, „gegen den Hintergrund gesetzlich gesicherter Rechte den Terror in seiner furchtbaren Willkür und Gesetzlosigkeit desto schärfer erkennen zu lassen“. 
repressive Staat ständig zu kämpfen hatte ${ }^{13}$. Der Partei- und Staatsapparat war damit bestrebt, einen neuen Menschen zu züchten, „der aus dem Laboratorium der Revolution hervorging“" (BABEROWSKi 2014: 134), den Regeln des dogmatischen Marxismus folgte und als Kämpfer gegen innere und äußere Feinde in den Krieg zog. Im Prozess der proletarischen Selbstwerdung wurde er gezwungen, an die unbegrenzten Möglichkeiten der menschlichen Natur und die Idee der fortschreitenden, bolschewistischen Weltrevolution zu glauben, zu der es keine Alternative im sozialpolitischen und kulturellen Alltag gab.

Die sozialistischen Arbeiter, denen man alle Freiheiten kollektiv entzog (vgl. ARENDT 2015: 691), wurden in eine gigantische Zwangsarbeiterschaft verwandelt und je nach Erfüllung der festgesetzten Arbeitsnormen bewertet; auch freie Bauern mussten ihre Bauernhöfe aufgeben und den Kolchosen beitreten. Die Kollektivierung der 20er-Jahre machte Bauern zu Sklaven, über die die bolschewistischen Machthaber nach Belieben verfügen konnten. Mit der Zeit wütete der Staatsterror überall; „,er traf nicht nur Kommunisten und Generäle, sondern friß sich durch alle Schichten der sowjetischen Gesellschaft" (BABEROwsKi 2014: 317) und bedeutete meistens den Tod für die Betroffenen.

Die Gewalt im Sowjetstaat war „ein unverzichtbares Instrument der Disziplinierung und Umerziehung“ (BABEROwsKi 2014: 14) von Sowjetmenschen, die "nach Belieben stigmatisiert, in Angst und Schrecken versetzt oder getötet werden“ (BABEROwSKI 2014: 14) konnten. Die Zerstörungswut hat letztlich dazu geführt, dass die kommunistische Partei sich selbst zerstörte, als die Zeit der Großen Säuberung (1936-1938) eintrat und der gesellschaftspolitischen und kulturellen Destruktion ${ }^{14}$ dann keine Grenzen mehr gesetzt wurden.

\subsection{Wolgadeutsche Presse}

Die deutschsprachige Presse ${ }^{15}$ in der Wolgarepublik (vgl. MęDELSKa 2014: 219-243; Cieszkowski 2017: 11-16), die in den 20er- und 30er-Jahren "national nach der Form, dem Inhalt nach sozialistisch" (ENGEL-BRAUNSCHMIDT 1987: 115) war, wurde nach dem Vorbild der sowjetischen Presse gestaltet, die zentral organisiert war; sie hatte einen popularisierend-propagandistischen Charakter und diente der Partei dazu, die Idee einer kommunistischen Gesellschaft umzusetzen und die dazu erforderliche Agitationsarbeit unter den Volksmassen zu fördern. Dies betrifft nicht nur die Berichterstattung in der Tagespresse, sondern auch zwei deutschsprachige Fachzeitschriften - die „Bauernzeitung“(1921), die kurz nach ihrer Gründung in „Unsere Wirtschaft“, sowie das „Wolgadeutsche Schulblatt“ (1922), das anfangs in „Zur Neuen Schule“ (1925-1927) und schließlich in „Revolution und Kultur“ (1930-1935) umbenannt wurden; mit den beiden Titeln der Fachpresse lässt sich außerdem sehr gut die Tendenz zur mehrmaligen Umbenennung der Pressetitel exemplifizieren.

13 Das sozial, politisch und kulturell determinierte Bild des sowjetischen Feindes wird bei BABEROwSKI (2014: 154-172) protokolliert.

14 BABEROWSKI (2014: 304-308) spricht sogar von der Selbstzerstörung des Staatsapparats.

15 Näheres zum wolgadeutschen Pressediskurs vor 1917 vgl. CieszKowski (2015: 45-46). 
Auch das Pressevertriebssystem war ideologisch bedingt. Einerseits ist man dem territorialadministrativen Prinzip gefolgt, indem man Zentral- und Bezirkspresse gründete, andererseits wollte man zentral bestimmen, welche Presse von welchen Bevölkerungsgruppen zu lesen war (vgl. (ИААрионова 2006: 74). Noch in der ersten Hälfte der 20er-Jahre wollte man die Zahl der deutschsprachigen Pressetitel erhöhen; dann musste man aus finanziellen Gründen die Zahl der Ausgaben und deren Umfang verringern, manche Zeitungen hat man sogar geschlossen

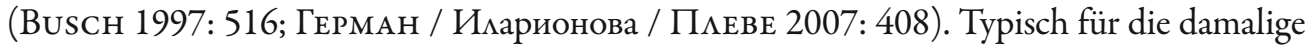
Presse ist die Mehrfachvergabe von Titeln (z. B. fünf Presseorgane mit dem Titel „Lenins Weg“).

In der Lokalpresse wurden neben Nachrichten aus der jeweiligen Region, etliche Artikel aus der Zentralpresse abgedruckt, u. a. Ansprachen von Stalin und anderen prominenten Staats- und Parteifunktionären, Verpflichtungen von Arbeitsbrigaden der Großbetriebe oder Kolchose, aktuelle Daten zum sozialistischen Wettbewerb, Informationen über den Verlauf verschiedener politischer und wirtschaftlicher Kampagnen sowie Berichte über die Beschlüsse der einzelnen Staats- und Parteiorgane; engagiert dazu wurden Arbeiter- und Bauernkorrespondenten, deren Fähigkeiten auf dem Gebiet der Schreibkunst meistens defizitär waren. Schreibinkonsequenzen und auftretende grammatisch-stilistische Fehler sind eins der sprachlichen Charakteristika der damaligen deutschsprachigen Pressetexte in der Wolgarepublik ${ }^{16}$.

Auf der Grundlage der deutschsprachigen Presse, deren Bestände in russischen Bibliotheken und Archiven heute wieder zugänglich sind, war es denkbar, die wolgadeutsche Lexik der 20er- und 30er-Jahre zu erfassen und lexikologisch / lexikographisch zu bearbeiten; im Folgenden wird sie exemplarisch zur Illustration der wolgadeutschen Sprachspezifik genutzt ${ }^{17}$.

\subsection{Wolgadeutsche Sprachspezifik}

Der wolgadeutsche Wortkatalog dieser Zeit besteht neben deutscheigenen Spracheinheiten (die aus meinen Überlegungen aus wohl verständlichen Gründen ausgeschlossen werden) aus zwei Gruppen von russischen Lexemen: zum einen gibt es Lexeme, die bereits vor 1917 in den Sprachgebrauch der Wolgadeutschen eingegangen sind (z. B. aмбар - Ambar; кyлaк - Kulak; $n y \partial-P u d)$, zum anderen haben wir mit russischen Lexemen zu tun, die nach 1917 entweder in Vollform angeeignet wurden (z. B. мaсcовик-Massowik ${ }^{18}$; nредседатель-Praedsidatel; ударник - Udarnik ${ }^{19}$ ) oder nach dem Vorbild des Russischen unter Ausnutzung der aktiven deutschen Wortbildungsmuster entstanden sind (z. B. Aнтирождество - Antiweihnachten; nросвещенещ - Politischer Aufklärungsarbeiter ${ }^{20}$; nрофсоюз - Profverband).

Die neue Lexik dient dazu, sich mit der politisch-wirtschaftlichen und kulturellen Realität in der Wolgarepublik auseinanderzusetzen, auf die Organisierung und Lenkung

16 Näheres zum wolgadeutschen Pressediskurs nach 1917 vgl. CieszKowski (2015: 47-49).

17 ADabei beziehe ich mich auf ausgewählte Beispiele aus der Korpusdatei, die ich im Rahmen des Forschungsprojekts Zur Sowjetisierung der wolgadeutschen Sprachvarietät in den 20er-und 30er-Jahren zusammengestellt habe (vgl. CIESZKOWSKI 2017: 14-16).

18 Parallel dazu auch Massenarbeiter.

19 Parallel dazu auch Stoßbrigadler.

20 Parallel dazu auch Politproswetarbeiter. 
gesellschaftlicher Prozesse einzuwirken und damit verbundene Wertungen zu vermitteln. Die Lexik ist klassen- und ideologiegebunden; mit deren Hilfe werden ideologische und weltanschauliche Positionen festgehalten, politische Einstellungen und Einsichten geäußert sowie die Zugehörigkeit zu diversen Klassen, Schichten, Parteien und gesellschaftlichen Organisationen bestimmt (vgl. SCHIPPAN 1984: 252).

In der wolgadeutschen Lexik spiegelt sich der in den 20er- und 30er-Jahren fortschreitende Prozess der Sowjetisierung wider, in dem etlichen Erscheinungen und Ereignissen, Vorgängen und Institutionen des gesellschaftlichen, politischen und kulturellen Lebens sowie alltäglichen Räumen menschlicher Aktivitäten spezifische, sprachliche Formen verliehen werden, die sie damals als typisch sowjetisch markiert haben und heute als typisch sowjetisch identifizieren lassen.

Als dominierende sprachliche Tendenzen im wolgadeutschen Wortgebrauch sind folgende zu nennen:

1.

Der Gebrauch typischer Lexik, mit der die Entwicklungen in der sowjetischen Gesellschaft bezeugt (u. a. Kollektivierung auf dem Lande, Organisierung der Arbeit in volkswirtschaftlichen Betrieben, die Liquidierung des Analphabetentums, die Partei- und Nationalitätenpolitik usw.) und als Ergebnis sprachlicher Auseinandersetzungen mit der politisch-wirtschaftlichen und kulturellen Realität im Sowjetstaat zu werten ist; meistens sind sie - aus der Perspektive der Wortbildung gesehen - Simplizia; zum Beispiel:

Die Aufgaben der Organisierung der Stachanow-Saat erfordern, daß die Sowjets als Organe der Diktatur des Proletariats im Dorfe, ihre Rolle als Organisatoren der Massen zu bolschewistischer Verwirklichung der von Genossen Stalin bezüglich der entschiedenen Steigerung der Ernteerträge, heben (STG ${ }^{21}$ 10/1936, S. 1).

Nur die Diktatur des Proletariats unter der Leitung der großen Partei Lenins-Stalins, nur die LeninStalinsche Nationalitätenpolitik gab den deutschen Werktätigen die Möglichkeit, ihre Exploitatoren zu vertreiben, die kulakischen Nationalisten zu zertrümmern und eine Kultur national nach der Form, sozialistisch dem Inhalte nach zu schaffen (STG 20/1936, S. 1).

Aber auch auf dem Gebiete des sozialistischen Aufbaus, der sozialen Umgestaltung der Landwirtschaft, des Kampfes gegen das Analphabetentum und die Trunksucht usw. wollen die Pioniere nicht hinter den erwachsenen Werktätigen zurückbleiben (DT 16/1929, S. 2).

Es muß enge Verbindung mit der Prokuratur, der Redaktion „Stalinez“, den Wandzeitungen hergestellt, die Wachsamkeit gegenüber den noch nicht überlebten Gesetzverletzer, den Mißständen in den Kolchosen, Sowchosen und Kooperativen behoben und verstärkt werden (STG 62/1935, S. 2).

2.

Die Tendenz zur Reihenbildung, deren Folge Serien von Determinativkomposita sind, in denen ,als erste oder zweite Konstituente massenhaft das gleiche Element erscheint, ohne daß von Suffix- oder Präfixbildungen gesprochen werden kann" (Fleischer 1976: 102); solche

21 Aufösung der abgekürzt zitierten Pressetitel befindet sich am Ende des Aufsatzes. 
Bildungen entsprechen russischen Wortgruppen, die politisch-ideologisch aufgeladene Wörter enthalten; diese Tendenz steht im Gegensatz zur früher erwähnten Tendenz:

(a) reihenbildend ist die erste Konstituente:

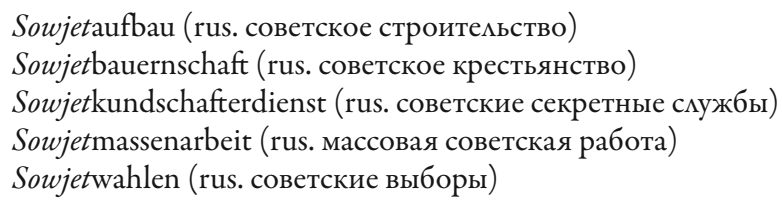

Andere reihenbildende Konstituenten, die zahlenmäßig stark in Erscheinung treten, sind u. a.: Brigaden-, Combine-, Getreide-, Kanton-, Klasse-, Kolchos-, Kollektiv-, Komsomol-, Kulak-, Partei-, Pionier-, Rayon- und Stachanow-.

(b) außerdem kann die zweite Konstituente reihenbildend sein:

Einheitsfront (rus. единый фронт)

Erntefront (rus. уборочный фронт)

Getreidebeschaffungsfront (rus. хиебозаготовительный фронт)

Kolchosfront (rus. колхозный фронт)

Kulturfront (rus. культурный фронт)

(c) reihenbildend wirken auch manche Wortgruppen bei der Bildung von Komposita:

Arbeiter- und Bauernarmee (rus. Рабоче-Крестьянская Красная Армия)

Arbeiter- und Bauernbewegung (rus. рабоче-крестьянское Авижение)

Arbeiter- und Bauerninspektion (rus. Рабоче-крестьянская инспекция)

Arbeiter-und Bauernmiliz (rus. рабоче-крестьянская мимиция)

Arbeiter- und Bauernkinder (rus. рабоче-крестьянские дети)

(d) auch Simplizia wirken reihenbildend in festen Wortgruppen:

Rote Ecke (rus. красный уголок)

Rote Fahne (rus. красный фмаг)

Rote Hauptstadt (rus. красная столица)

Roter Zug (rus. красный обоз)

Rote Tafel (rus. красная доска)

3.

Abkürzungen und Kurzwörter, die die Zeit des Bestehens der Wolgarepublik stigmatisieren, nach russischem Wortbildungsmuster Glied für Glied übersetzt (Kalkierung) oder vollständig übernommen wurden und nur vereinzelt in den allgemeinen Sprachgebrach (vorzugsweise in der ehemaligen DDR $)^{22}$ eingegangen sind.

22 Solche Struktureinheiten werden in der folgenden Übersicht mit Sternchen versehen. 
ABI* (Arbeiter- und Bauerninspektion, rus. Рабоче-крестьянская инспекция; РКИ)

Kultprop (Kulturpropaganda, rus. культпроп, культурная пропаганда)

MTS* (Maschinen-Traktorenstation, rus. машинно-тракторная станция; МТC)

Polit-Tag (Tag der politischen Arbeit, rus. политдень, политический день)

Rabfak (Arbeiterfakultät ${ }^{23}$, rus. рабфак, рабочий факультет)

Auch manche Abkürzungen wirken reihenbildend:

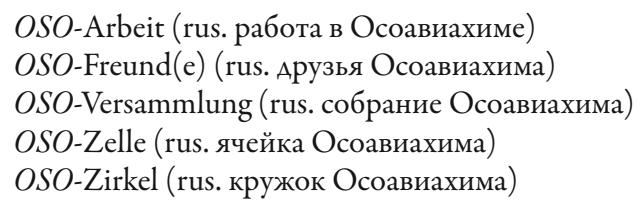

4.

Aufkommen von femininen Berufsbezeichnungen mit dem Suffix -in, die vom maskulinen Formen im Prozess der Motion (Movierung) (vgl. Fleischer 1976: 182-185) abgeleitet werden. Sie versprachlichen eine durchaus ideologisch bedingte und im sozialen Umfeld von der Partei ordinierte Gleichberechtigung der Frauen in der Sowjetunion.

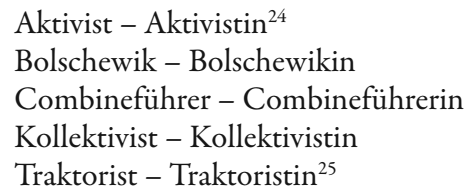

5.

Hybride Sätze, in denen neben deutschen Strukturelementen auch russische Einschübe (als wichtige Bedeutungseinheiten, meistens Substantive) enthalten sind:

Auch die Stallung ist nicht in Ordnung, die Kletken sind zusammengebrochen, was zur unplanmäßigen Befruchtung der Schweine führt (SWE 2/1938, S. 2).

Eine merkwürdige Smitschka habt hier eingeführt. Eine umgekehrte Verschmelzung von Stadt und Land (...) (DK 7(3)1935, S. 37).

Und dann die Freude über das Licht und das Artel, wie es mit einem Schlage anders aussieht. Und auch die besten Udarniki kommen aufs Bild (DK 5-6 (3)1935, S. 28).

Bis jetzt sind noch Fälle von Nichtumfassung aller schulpflichtigen Kinder zu verzeichnen (Wodjanka) (KWSB 9(5)1938, S. 2).

\footnotetext{
23 In der DDR als Arbeiter- und Bauernfakultät (ABF) bekannt.

24 Parallel dazu auch Frauenaktivist.

25 Parallel dazu auch Frauentraktorist.
} 
SELENSKI organisierte im Zentrosojus und im System der Konsumkooperation Schädigungsgruppen (...) (STG 28-29 / 1938, S. 2).

6.

Exotismen als lexikalische Einheiten, die aus verschiedenen Sprachen der das sowjetische Territorium bewohnenden nationalen Minderheiten stammen; z. B.:

Wissen die Kinder und Pioniere, daß die russischen Zaren im Kaukasus die selbstbewußten Bergbewohner „bändigte“, indem sie viele Dörfer und Ortschaften (Auls) dem Erdboden gleich machten, das Eigentum vernichteten und die Leute umbrachten (DT 24/1929, S. 9)?

Das Land für Bachtschu ist geackert und geeggt, wird aber noch nicht eingesät - da noch Frost eintreten kann (STG 14/1936, S. 1).

Ob es Schmuggler sind? Oder Basmatschen (DK 2(6)1938, S. 5)?

Die Kolchosverwaltung verpflichten, das Bedienungspersonal der Schweine mit Chalats und Gummigaloschen zu versorgen (RF 116/1937, S. 2).

Diesen Sommer, während der Ferien, besuchte ich wieder meine Staniza, wo ich geboren, meine Kindheit verlebt, arbeiten gelernt (SB 138/1937, S. 2).

Die wolgadeutsche Varietät der deutschen Sprache hat in den 20er- und 30er-Jahren des 20. Jh. einerseits den ideologiegebundenen Wortschatz des Russischen aufgenommen, andererseits potentielle Wortbildungsmöglichkeiten des deutschen Sprachsystems genutzt (darunter Komposita mit reihenbildenden Konstituenten, Movierungen als Ableitungen, Abkürzungen und Kurzwörter), um sprachlichen Notwendigkeiten des Alltags in einem kommunistisch regierten Staat zu entsprechen. Mit der aus der wolgadeutschen Presse exzerpierten Lexik lässt sich relativ unproblematisch beweisen, welche Strukturen darin bevorzugt wurden. Unbeantwortet muss aber die Frage bleiben, in welchem Ausmaß die wolgadeutsche, geschriebene Variante ein Teil des wolgadeutschen Sprachgebrauchs war und die gesprochene, mundartlich geprägte Variante des Deutschen in den Wolgakolonien mit beeinflusst hat.

\section{Literatur}

ARENDT, Hannah ( $\left.{ }^{18} 2015\right)$ : Elemente und Ursprünge totaler Herrschaft. Antisemitismus, Imperialismus, totale Herrschaft. München, Berlin: Piper.

BABERowsKi, Jörg (2012): Verbrannte Erde. Stalins Herrschaft der Gewalt. München: Fischer.

BEREND, Nina (1998): Sprachliche Anpassung. Eine soziolinguistisch-dialektologische Untersuchung zum Rußlanddeutschen. Tübingen: Narr.

Bohmann, Alfred (1970): Menschen und Grenzen. Bd. 3: Strukturwandel der deutschen Bevölkerung im sowjetischen Staats- und Verwaltungsbereich. Köln: Wissenschaft und Politik.

Brandes, Detlef (1997): Einwanderung und Entwicklung der Kolonien. In: STRICKER, Gerd (Hg.): Deutsche Geschichte im Osten Europas. Rußland. Berlin: Siedler, 35-110.

Busch, Margarete (1997): Presse in der Zwischenkriegszeit: Meinungsfreiheit oder Marionettentheater? In: Stricker, Gerd (Hg.): Deutsche Geschichte im Osten Europas. Rußland. Berlin: Siedler, 515-519. 
Cieszkowski, Marek (2015): Zur Sowjetisierung der wolgadeutschen Sprachvarietät in den 20erund 30er-Jahren. In: Studia Germanica Gedanensia 33, 40-55.

CieszKowski, Marek (2017): Sowietyzacja Niemców rosyjskich w latach 20. i 30. XX wieku. Tom 3: Wczesny sowiecki jezyk niemiecki (na podstawie prasy, wydawnictw ksiażkowych i materiatów propagandowych wydawanych w Zwiąku Sowieckim) [Die Sowjetisierung der Sprache der Russlanddeutschen in den 20-er und 30-er Jahren des 20. Jh. Bd. 3: Frühes sowjetisches Deutsch]. Bydgoszcz: Wydawnictwo UKW.

DeIBERT, Edgar (2009): Sowjetdeutsche zwischen Hitler und Stalin. Deutsche in der UdSSR 1900-1950. Marburg: tectum.

Dinges, Georg (1923): Über unsere Mundarten. In: Beiträge zur Heimatkunde des deutschen Wolgagebiets. Mit einer Karte und einer Tabelle. Pokrowsk (Kosakenstadt): Abteilung für Volksbildung des Gebiets der Wolgadeutschen, 60-72.

Dietz, Barbara / Hilkes, Peter (1992): Rußlanddeutsche: Unbekannte im Osten. Geschichte - Situation - Zukunftsperspektiven. München: Olzog.

Domaschnew, Anatoli (1994): Einige Bemerkungen zum Begriff „Sprachinsel“ und zur Erforschung der rußlanddeutschen Mundarten. In: Berend, Nina / Mattheier, Klaus J. (Hg.) (1994): Sprachinselforschung. Eine Gedenkschrift für Hugo Jedig. Frankfurt/M.: Lang, 165-177.

Dulson, Andrej (1933): Einige lautliche Eigentümlichkeiten der wolgadeutschen Mundarten. In: Revolution und Kultur 5, 46-54.

Engel-BraunschmidT, Annelore (1987): „National nach der Form, dem Inhalt nach sozialistisch”: zur besonderen Situation der deutschen Literatur in der Sowjetunion. In: Kappeler, Andreas / Meissner, Boris / Simon, Gerhard (Hg.): Die Deutschen im russischen Reich und im Sowjetstaat. Köln: Marcus, 115-135.

FleisCHeR, Wolfgang (1976): Wortbildung der deutschen Gegenwartssprache. Leipzig: Bibliographisches Institut.

FrANK, Helene (1992): Zur sprachlichen Entwicklung der deutschen Minderheiten in Rußland und in der Sowjetunion. Frankfurt/M.: Lang.

Jedig, Hugo H. (1986): Die deutschen Mundarten in der Sowjetunion. In: Uhlisch, G. (Hg.): Das Wort. Germanistisches Jahrbuch DDR-UdSSR. Zwickau: Zentralstelle, 74-80.

JEDIG, Hugo H. (2014): Über den sprachlichen Nachlass der Saratower dialektologischen Schule im Archiv. In: Jedig, Hugo H. (2014): Lepel, Laumptje, Lostichkeit. Herausgegeben von Nina BEREND. Mannheim: Institut für Deutsche Sprache, 155-166.

LEBIODA, Tadeusz (2004): Niemcy rosyjscy w polityce RFN na tle ich znaczenia $w$ Rosji i relacjach niemiecko-rosyjskich $w$ latach 1763-2003 [Russslanddeutsche in der Politik der BRD vom Hintergrund deren Bedeutung in Russland und die deutsch-russischen Beziehungen in den Jahren 1763-2003]. Wrocław: Arboretum.

LÖFfLER, Heinrich (1994): Germanistische Soziolinguistik. Berlin: Erich Schmidt.

MęDELSKA, Jolanta (2014): Prasa radziecka wydawana w latach 30. XX w. w Republice Niemców Powołża [Sowjetische Presse in den 30er-Jahren des 20. Jh. in der Republik der Wolgadeutschen]. In: Acta Baltico-Slavica. 38, 219-243.

MęDELSKa, Jolanta / Cieszkowski, Marek / Jankowiak-Rutkowska, Maria (2017): Sowietyzacja Niemców rosyjskich w latach 20. i 30. XX wieku. Tom 1 (część 1): Zbiorowość niemiecka na ziemi rosyjskiej (od zarania do początków XX wieku) [Die Sowjetisierung der Sprache der Russlanddeutschen 
in den 20-er und 30-er Jahren des 20. Jh. Bd. 1 (T. 1): Deutsche Bevölkerungsgruppen auf russischem Gebiet (von den Anfängen bis zum Beginn des 20. Jh.)]. Bydgoszcz: Wydawnictwo UKW. Mędelska, Jolanta / Cieszkowski, Marek / SobCZak, Michał (2017): Sowietyzacja Niemców rosyjskich w latach 20. i 30. XX wieku. Tom 1 (część 2): Losy Niemców rosyjskich w latach 20. i 30. XX wieku [Die Sowjetisierung der Sprache ... Bd. 1 (T. 2): Das Schicksal der Russlanddeutschen in den 20-er und 30-er Jahren des 20. Jh.]. Bydgoszcz: Wyd. UKW.

Moskaljuk, Larissa (1998): Tradition und Dynamik im Bereich der Alltagslexik der rußlanddeutschen Dialekte. In: RetTERATH, Hans-Werner (Hg.): Wanderer und Wanderinnen zwischen zwei Welten? Zur kulturellen Integration russlanddeutscher Aussiedlerinnen und Aussiedler in der Bundesrepublik Deutschland. Freiburg im Breisgau: Johannes-Künzig-Institut für Ostdeutsche Volkskunde, 27-35.

Neutatz, Dietmar (2016): Wo steht die Forschung über die Russlanddeutschen. In: Eisfeld, Alfred (Hg.): Deutsche im Schwarzmeergebiet, auf der Krim und im Kaukasus vom 19. Jahrbundert bis 1941. Hamburg: Kovač, 595-612.

Schippan, Thea (1984): Lexikologie der deutschen Gegenwartssprache. Leipzig: Bibliographisches Institut.

SCHIRMUNSKI, Viktor (1928): Die deutschen Kolonien in der Ukraine. Moskau: Zentraler Völkerverlag der Sowjetunion.

Sinner, Peter (1923): Kurzgefaßte Geschichte der deutschen Wolgakolonien. In: Beiträge zur Heimatkunde des deutschen Wolgagebiets. Mit einer Karte und einer Tabelle. Pokrowsk (Kosakenstadt): Abteilung für Volksbildung des Gebiets der Wolgadeutschen, 5-28.

SCHULze-MöLKaU, Rudolf (1931): Die Grundzüge des wolgadeutschen Staatswesens im Rahmen der russischen Nationalitätenpolitik. München: Ernst Reinhardt.

Герман, Аркадий А. / Иларионова, Татьяна С. / Пиеве, Игор Р. (2007): История немцев России. Учебное пособие. Москва: МСНК-пресс.

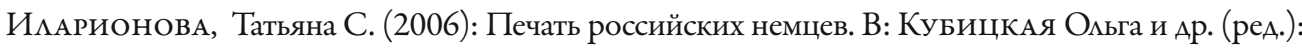
Немцы России: энщиклопедия. Т. 3. Москва: Общественная Академия наукроссийских немщев, 67-76.

\section{Auflösung der abgekürzt zitierten Pressetitel}

DK - Der Kämpfer. Organ des Bundes der Sowjetschriftsteller der ASSRdWD, Nr. 5-6, 7/1935, $2 / 1938$

DT - Die Trommel. Organ des Gebietskomitees des LKJV, des Gebietsbüros der Jungpioniere und des Volkskommissariats für Volksbildung der ASRR der Wolgadeutschen, Nr. 16, 24/1929

KWSB - Kollektivwirtschaftlicher Stoßbrigadler. Organ des Kantonkomitees der KP(B)SU und des Kanton-Vollzugskomitees des Gmelinkaer Kantons der ASSR der WD, Nr. 9/1938

STG - Stalinist / СТААИНЕЦ. Organ des Gnadenflurer Kantonkomitees der KP(B)SU und des Kantonvollzugskomitees, Nr. 62/1935, 10, 14, 20/1936 
SWE - Stalins Weg. Organ des Kantonparteikomitees der KP(B)SU, des Kantonvollzugskomitees des Erlenbacher Kantons der Autonomen Sozialistischen Sowjetrepublik der Wolgadeutschen, Nr. 2/1938

RJ - Rote Jugend. Organ des Gebietskomitees des LKJVdSU und des Engelser Stadtkomsomolkomitees der ASSR der Wolgadeutschen, Nr. 2-3/1934 